\title{
Qualidade higiênico-sanitária dos restaurantes cadastrados na Vigilância Sanitária de Santa Maria, RS, Brasil, no período de 2006 a 2010
}

\author{
Hygienic-sanitary status of restaurants recorded in the health surveillance unit of Santa Maria, Rio \\ Grande do Sul, Brazil, from 2006 to 2010
}

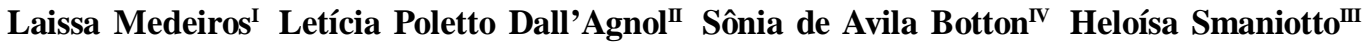 \\ Rafael Potter ${ }^{\text {III }}$ Marli Matiko Anraku de Campos ${ }^{\text {V }}$ Karen Mello de Mattos $^{\mathrm{I}}$ Luis Felipe Dias Lopes $^{\text {VI }}$ \\ Luís Antonio Sangioni ${ }^{\mathrm{N}^{*}}$
}

RESUMO

O presente estudo teve por objetivo avaliar, através dos roteiros de inspeção, as condições higiênico-sanitárias dos restaurantes cadastrados pela Vigilância Sanitária (VISA) do município de Santa Maria - RS. Neste estudo, foram incluídos 315 roteiros de vistorias provenientes de 120 restaurantes com alvarás sanitários e fiscalizados no período de janeiro de 2006 a dezembro de 2010. As variáveis (a) condições de armazenamento dos alimentos, (b) equipamentos de conservação a frio e a quente, (c) estrutura física do estabelecimento e (d) condições higiênico-sanitária dos manipuladores dos alimentos foram compiladas e avaliadas. Os resultados foram analisados estatisticamente pelo programa SAS (Statistical Analysis System) versão 9.2 e submetidos aos testes de Qui-quadrado e Fischer. Constatou-se que, no ano de 2009, ocorreram os maiores índices de desconformidades legais nos aspectos higênico-sanitários nos estabelecimentos pesquisados. Esse fato pode estar relacionado ao aumento do número de fiscais sanitários contratados, à descentralização do setor de fiscalização, o que possibilitou a realização de um maior detalhamento dos requisitos fundamentais a serem avaliados nos roteiros de vistoria e, consequentemente, havendo um aumento nas notificações oficiais. Além disso, no ano de 2010, os estabelecimentos apresentaram melhores índices de adequação às boas práticas de produção de alimentos. Com base nas informações obtidas, verificou-se que os serviços prestados pelo setor de alimentos da VISA de Santa Maria estão em crescente evolução.
Palavras-chave: alimentos, saúde pública, epidemiologia, inspeção sanitária.

\begin{abstract}
The present study was to evaluate, through the forms of inspection, the sanitary conditions of restaurants registered for Sanitary Surveillance (VISA) in the municipality of Santa Maria, RS, Brazil. This study included 315 inspection forms, from 120 restaurants with sanitary permits from January 2006 to December 2010. The following variables: (a) food storage conditions, (b) equipment for hot and cold storage, (c) the physical structure of establishment and (d) hygienic and sanitary conditions of food handlers, were compiled and evaluated. The results were statistically analyzed by SAS (Statistical Analysis System) version 9.2 and submitted to the chi-square and Fischer tests. It was observed that in 2009 there were higher rates of nonconformities in the legal hygienic and sanitary aspects in the establishments studied. Such fact can be related to the increasing of the number of health inspectors hired, decentralization of surveillance division, what allowed the achievement of a more detailed inspection forms, and consequently there is an increase in official reports. Additionally, in 2010, food establishments had better rates of conformity with food good manufacturing practices. Based on the information obtained, it was found that the services provided by the VISA of Santa Maria, RS, Brazil are in increasing development.
\end{abstract}

Key words: food, public health, epidemiology, health inspection.

\footnotetext{
'Programa de Educação para o Trabalho (PET), Vigilância Sanitária (VISA), Centro Universitário Franciscano (UNIFRA), Santa Maria, RS, Brasil.

"IPET, VISA, Universidade Federal de Santa Maria (UFSM), Santa Maria, RS, Brasil.

IIIVigilância em Saúde, VISA, Prefeitura Municipal de Santa Maria, Santa Maria, RS, Brasil.

IV Departamento de Medicina Veterinária Preventiva, PET, VISA, UFSM, Santa Maria, RS, Brasil. E-mail: lasangioni@gmail.com.

*Autor para correspondência.

Departamento de Análises Clínicas e Toxicológicas, PET, VISA, UFSM, Santa Maria, RS, Brasil.

VIDepartamento de Ciências Administrativas, PET, VISA, UFSM, Santa Maria, RS, Brasil.
} 


\section{INTRODUÇÃO}

A segurança alimentar é um desafio atual à saúde pública, uma vez que visa a oferecer alimentos inócuos aos cidadãos e a manter a integridade da saúde do consumidor (PILLA, 2009). Dentre os principais fatores relacionados à ocorrência de Doenças de Origem Alimentar (DTAs), destacam-se: más condições de higiene na manipulação, no armazenamento e na conservação dos alimentos; uso incorreto do binômio tempo-temperatura; falta de adequação e conservação da estrutura física dos estabelecimentos; entre outros (SILVA, 2010).

De acordo com BENEVIDES \& LOVATTI (2004), as DTAs podem ocasionar altas taxas de mortalidade em indivíduos expostos. Isso dependerá de fatores como: a ingestão de alimento de má qualidade contendo agentes infecciosos ou toxinas; a quantidade do alimento contaminado ingerido; o estado imunológico do indivíduo acometido; etc. Os erros mais frequentes na manipulação dos alimentos encontrados incluem: o preparo dos alimentos com muita antecedência; o cozimento, o armazenamento e/ou o reaquecimento das refeições de forma inadequada; a contaminação cruzada de micro-organismos e toxinas; bem como a má-higiene pessoal dos operadores (ABREU et al., 2011).

O objetivo da Vigilância Sanitária (VISA) em relação aos alimentos é fiscalizar, licenciar e cadastrar os estabelecimentos que produzem, comercializam, distribuem e/ou armazenam alimentos; bem como a fiscalização do transporte dos produtos alimentícios (GERMANO \& GERMANO, 2011). A inspeção sanitária é o procedimento de fiscalização realizada pela autoridade sanitária que analisa toda a cadeia produtiva através das Boas Práticas de Fabricação (BPF), definindo parâmetros de qualidade e segurança, com a regulamentação dos procedimentos, baseados no sistema de Análise de Perigos e Pontos Críticos de Controle (APPCC). As BPFs de alimentos consistem em avaliar nos estabelecimentos: as instalações, os equipamentos e os utensílios; a tecnologia de produção empregada; as formas de controle de qualidade; os procedimentos de desinfecção e controle de roedores e vetores; o armazenamento, o transporte, a comercialização dos produtos; bem como o monitoramento da saúde dos funcionários (SILVA, 2010).

Nesse contexto, este estudo teve por objetivo avaliar as condições higiênico-sanitárias dos restaurantes, cadastrados na VISA, de Santa Maria/ RS, durante o período de 2006 a 2010.

\section{MATERIAL E MÉTODOS}

Este estudo teve um delineamento transversal, com coleta de dados secundários provenientes dos roteiros de vistorias nos restaurantes cadastrados pela VISA em Santa Maria, RS. Incluiu-se neste estudo a análise dos alvarás dos serviços de alimentação vistoriados no período de janeiro de 2006 a dezembro de 2010 e seus respectivos roteiros de inspeção sanitária. A coleta de dados ocorreu nos meses de março e abril de 2011. Foram compiladas e avaliadas as seguintes variáveis: (a) condições de armazenamento dos alimentos, (b) equipamentos de conservação a frio e a quente, (c) estrutura física do estabelecimento e (d) condições higiênico-sanitária dos manipuladores dos alimentos.

No item armazenamento dos alimentos, foram pesquisados: as condições das embalagens, as formas de armazenamento, a validade e registro dos produtos alimentícios e as possibilidades de reaproveitamento dos alimentos. Em relação aos equipamentos de armazenamento e de exposição dos alimentos, foram avaliadas as estruturas físicas e a manutenção periódica. $\mathrm{Na}$ área dos estabelecimentos, foram verificadas as condições físicas e de conservações das construções, incluindo as portas, janelas, forros, paredes, pisos, iluminação e ventilação. Em relação aos manipuladores de alimentos, foram analisados: a utilização dos uniformes, a higiene pessoal, a utilização de adornos e a existência de afecções cutâneas. Cada item analisado no roteiro de vistoria foi classificado em acordo ou desacordo, baseados na Portaria 78/2009 da Secretaria de Saúde do RS e na resolução RDC no 216/2004 da ANVISA, que foram utilizados como critérios legais para estabelecer as conformidades e/ou irregularidades dos restaurantes (BRASIL, 2004; RIO GRANDE DO SUL, 2009). Os resultados foram submetidos à análise estatística pelo teste do Qui-quadrado ou Teste Exato de Fischer com auxílio do programa SAS (Statistical Analisys System) versão 9.2, quando foram associadas às diferentes variáveis, com nível de significância de $5 \%$.

\section{RESULTADOS E DISCUSSÃO}

Neste estudo, 315 roteiros de vistorias provenientes de 120 restaurantes com alvarás sanitários e fiscalizados pelos inspetores sanitários da VISA foram analisados. O número de vistorias nos serviços de alimentação avaliados nesta pesquisa foi de $53(16,8 \%)$, $63(20 \%), 73(23,1 \%), 74(23,5 \%)$ e $52(16,5 \%)$ nos anos de 2006, 2007, 2008, 2009 e 2010, respectivamente. O resultado da avaliação das condições de armazenamento, incluindo as matérias-primas e 
ingredientes dos serviços de alimentação, está demonstrado na figura 1-a. Observou-se que houve um maior índice de desacordos no item alimentos armazenados corretamente, no ano de 2009, quando comparados com os demais anos (2009 e 2010; $\mathrm{P}=0,0053)$. As principais alterações observadas incluíram: alimentos armazenados sem invólucro, embalagens avariadas, uso de materiais inadequados para a embalagem. Salienta-se que, em todos os demais anos avaliados, grande parte dos estabelecimentos comerciais apresentava-se em conformidade com a resolução RDC n 216 (BRASIL, 2004).

As embalagens dos alimentos devem ser armazenadas em áreas destinadas para este fim e em perfeitas condições higiênico-sanitárias. Após a retirada da embalagem original, os alimentos industrializados que não tiverem sido totalmente utilizados, devem ser colocados em recipientes adequados e identificados (BRASIL, 2004). Preconiza- se o uso de materiais específicos para guardar os alimentos, tais como: vasilhames plásticos com tampa, saco plástico apropriado, filme de PVC transparente, entre outros (ABERC, 2009). Um estudo conduzido por CARDOSO et al. (2005) verificou que $40 \%$ das unidades de alimentação e nutrição utilizavam vasilhames plásticos ou de vidro e com tampa, para armazenar o produto restante; $15 \%$ armazenavam na própria lata; $25 \%$ utilizavam toda a lata após aberta e $20 \%$ não utilizavam enlatados. DAMASCENO et al. (2002) constataram que, embora a maioria dos estabelecimentos apresentassem uma capacidade de armazenamento adequada, uma parcela de $37,5 \%$ demonstraram deficiências que incluíram desde equipamentos de refrigeração superlotados, alimentos estocados em caixas de papelão ou embalagens plásticas inadequadas e, até mesmo, a manutenção de alimentos crus e cozidos armazenados conjuntamente. Um fator que possibilita este procedimento pode estar

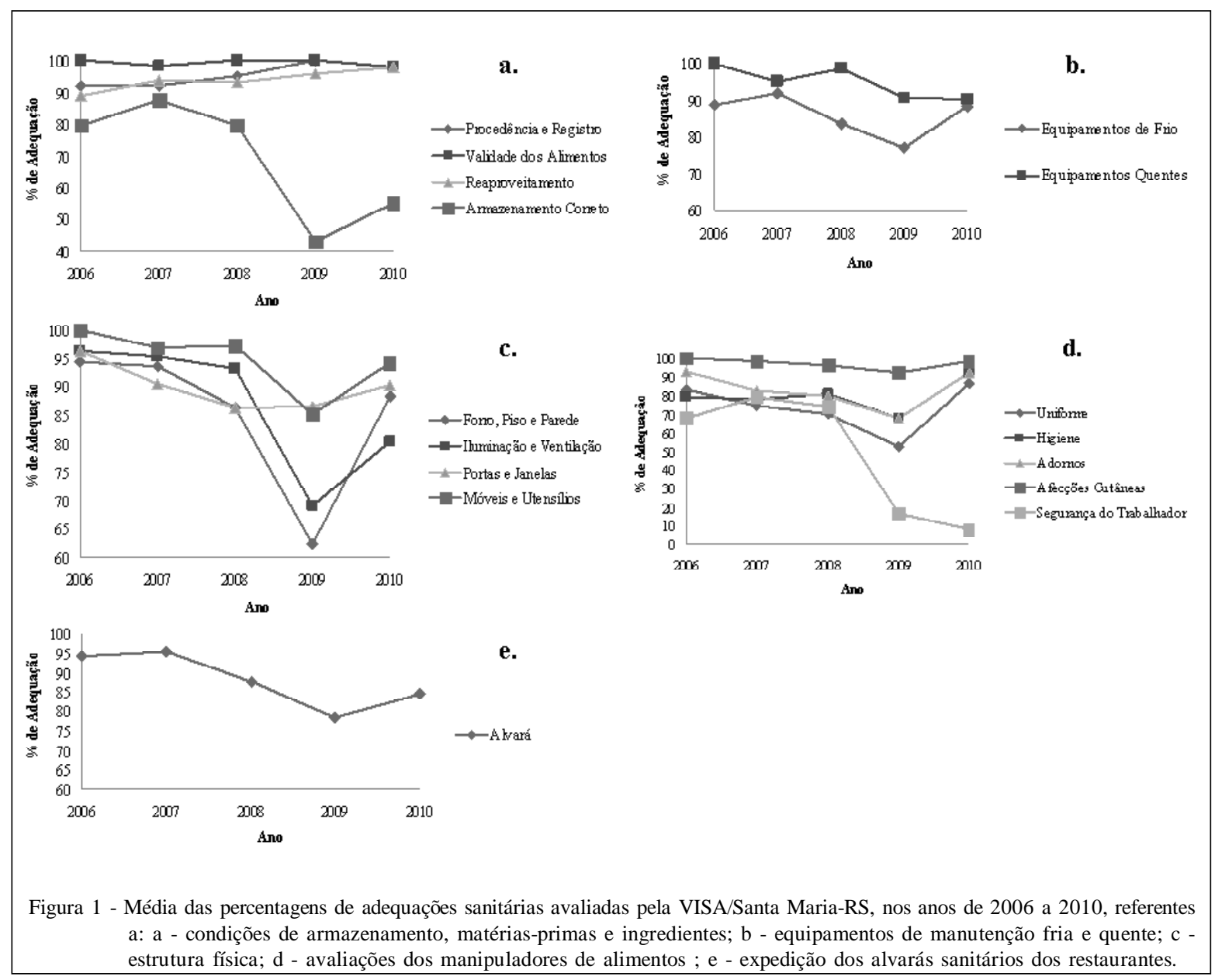

Ciência Rural, v.43, n.1, jan, 2013. 
relacionado à deficiência da capacitação contínua dos manipuladores de alimentos. No setor de alimentação, comumente, são contratados profissionais sem experiência na função que irão desempenhar, aliado ao baixo conhecimento sobre a manipulação higiênica dos alimentos, ignorando que descuidos nos procedimentos de preparo e armazenamento dos alimentos, também, podem constituir um modo de transmissão de doenças. Isso ressalta a necessidade de haver uma fiscalização sanitária bastante ativa e de caráter mais orientador do que punitivo (MELLO et al., 2010; PRAXEDES, 2003).

O resultado da avaliação dos equipamentos de manutenção fria e quente está apresentado na figura 1-b. O maior percentual de desconformidades legais nos equipamentos de manutenção fria e quente ocorreu no ano de 2009, quando comparados aos demais anos (2007 e $2009 \mathrm{P}=0,0198 ; 2009$ e $2010 \mathrm{P}=0,00409$, respectivamente). Um dos pontos críticos relevante nas BPF é o binômio tempo e temperatura, uma vez que a permanência de alimentos nas temperaturas consideradas como zona de perigo $\left(10^{\circ} \mathrm{C}\right.$ a $\left.60^{\circ} \mathrm{C}\right)$ promove a multiplicação rápida de micro-organismos, os quais poderão ocasionar danos à saúde do consumidor (STORCK \& DIAS, 2003). MURMANN et al. (2005) constataram que a maioria dos equipamentos frios destinados à conservação de alimentos em serviços de alimentação apresentava temperatura inadequada como sendo a principal irregularidade.

A figura 1-c apresenta os resultados avaliados em relação à estrutura física. Observou-se que os itens: forros, pisos e paredes; iluminação e ventilação; portas e janelas; e móveis e utensílios dos restaurantes pesquisados obtiveram o maior índice de inadequação no ano de 2009, em relação aos demais anos estudados (2009 e 2010; P= 0,0012). Alguns dos trabalhos realizados em vários estados brasileiros também denotaram este tipo de irregularidade. No estudo realizado por CASTRO (2007), foram analisados os aspectos sanitários em estabelecimentos localizados nos shoppings centers da cidade do Rio de Janeiro (RJ), onde foi observado algum tipo de avaria na sua estrutura física em 88,9\% dos locais visitados. Segundo MEZOMO (2002), a iluminação deve ser distribuída uniformemente pelo ambiente, devido à influência no comportamento das pessoas, uma vez que a utilização de uma iluminação adequada promove um aumento da visibilidade, possibilitando verificar as condições de limpeza do local e a inspeção adequada dos alimentos. GOLLUCKE et al. (2003) avaliaram as condições higiênico-sanitárias de restaurantes tipo selfservice de um município de São Paulo (SP), onde observaram que as condições de edificações apresentaram o menor índice de adequação. Esses resultados concordam com o estudo de DESCHAMPS et al. (2003) que avaliaram as condições higiênicosanitárias de cozinhas industriais instaladas no município de Blumenau (SC) e revelaram que, das 35 unidades visitadas, as condições físicas obtiveram os maiores índices de inadequações $(48,6 \%)$. MACHADO et al. (2009) avaliaram as condições higiênico-sanitárias de serviços de alimentação em Organizações Não Governamentais (ONG) de Toledo (PR) e constataram a presença de danos estruturais como: portas com fendas, tetos rachados e com infiltrações, além de paredes ásperas. Também as janelas, na maior parte dos locais, não apresentavam algum tipo de proteção, facilitando a entrada de roedores e/ou vetores. Além da importância da capacitação dos trabalhadores, outros fatores puderam influenciar nas inconformidades das condições higiênico-sanitárias, como as observadas neste estudo, que também foram descritas por MELLO et al. (2010), incluindo desde a estrutura física inapropriada até a falta de manutenção dos equipamentos e dos utensílios.

Os dados constantes na figura $1-d$ apresentam os resultados referentes às avaliações dos manipuladores, incluindo: higiene pessoal, afecções cutâneas, uniforme, uso de adornos e equipamentos de proteção individual (EPI) empregados para garantir a segurança do trabalhador. Novamente, em 2009, houve um maior percentual de inadequações quando comparados aos demais anos (2009 e 2010; $\mathrm{P}=0,0010)$. Ressalta-se que os uniformes utilizados pelos manipuladores de alimentos necessitam estar adequados às funções desempenhadas, além de estar limpos e em bom estado de conservação; ainda devem ser utilizados somente nas dependências internas do estabelecimento. A avaliação dos manipuladores também deve incluir as boas práticas de manipulação de gêneros alimentícios e o emprego de EPIs, tais como: a lavagem frequente das mãos; os banhos habituais; o corte e a higienização de unhas e cabelos; o emprego de cosméticos (esmalte, perfumes, maquiagem, talco, etc.) deve ser evitado durante a realização das atividades; os cabelos necessitam estar presos e envoltos por toucas; a proteção de ferimentos deve ser preconizada; o uso de óculos de proteção e calçados adequados à atividade desempenhada; a troca de uniforme (em bom estado de limpeza e conservação) necessita ser efetuada periodicamente, entre outros (BRASIL, 2004). Da mesma forma observada nesta pesquisa, outros estudos efetuados em diversos estados no Brasil constataram irregularidades com os manipuladores dos alimentos. No estudo de MIRANDA et al. (2002), ficou evidenciado que $41,7 \%$ dos manipuladores estavam com unhas grandes e sujas, 
$12,5 \%$ usavam adornos como anel e relógios e 58,3\% apresentavam afecções cutâneas nas mãos. BARBOSA NETA et al. (2004), em um restaurante da Universidade Federal do Rio Grande do Norte, observaram a falta de cuidados dos manipuladores de alimentos com as normas de higiene e EPIs, entre elas, destacaram-se: o uso de uniforme incompleto; a falta de limpeza dos calçados; as mãos e unhas mau higienizadas; a não utilização de luvas para manipulação dos alimentos; os cabelos, as barbas e os bigodes não estavam bem aparados e protegidos. No trabalho de WALKER et al. (2003), foi verificado que os manipuladores de alimentos demonstraram haver conhecimento dos principais aspectos relacionados à adequada higiene pessoal, assim como a não utilização de bijuterias e outros adornos durante a manipulação de alimentos. No entanto, AKUTSU et al. (2005) avaliaram a adequação das BPFs em serviços de alimentação e concluíram que a maioria dos locais analisados apresentou condições desfavoráveis em relação ao item manipuladores de alimentos. MELLO et al. (2010) e GUIMARÃES \& FIGUEIREDO (2010) verificaram que a frequência da higienização das mãos dos manipuladores de alimento era inadequada e, além disso, o uso de adornos (especialmente anéis, relógios e pulseiras) e esmalte nas unhas por esses profissionais durante o trabalho era efetuada. Outro item a ser destacado refere-se à saúde do trabalhador, pois conforme a $\mathrm{RDC} \mathrm{n}^{\circ} 216 / 2004$, o manipulador não pode apresentar feridas, lesões, chagas ou cortes nas mãos e braços, ou doenças de qualquer natureza como as gastroenterites. Além disso, os que estiverem acometidos de infecções pulmonares ou faringites devem ser afastados da manipulação de alimentos até a sua recuperação (BRASIL, 2004). Com isso, os resultados demonstrados neste estudo permitem inferir que os manipuladores podem ser veículos de DTAs, o que pode comprometer a saúde dos consumidores. Logo, para que sejam servidas refeições em condições adequadas para consumo, faz-se necessário que os manipuladores apresentem boa higiene pessoal (MELLO et al, 2010).

No período de 2006 a 2008, constatou-se que a média das qualidades das avaliações higiênicosanitárias dos restaurantes indicou que a grande maioria dos itens sanitários analisados estava de acordo com as normas vigentes. Entretanto, esta constatação não refletiu a realidade higiênico-sanitária da maioria dos estabelecimentos vistoriados. Na prática, houve, dentre outros fatores, a deficiência de notificações sanitárias neste período que pode ser atribuída à escassez de recursos humanos disponíveis para efetuarem os roteiros de inspeção. Consequentemente, havia um acúmulo de atribuições designadas a cada servidor que pode ter gerado a falta de rigor nas vistorias realizadas. Além disso, ações de capacitação e especialização visando a um serviço mais eficiente não ocorreram neste período. A partir do ano de 2008, com a descentralização dos serviços do setor de fiscalização da vigilância sanitária municipal, houve a possibilidade de realizar novas aquisições de funcionários e a promoção de cursos de capacitação dos servidores. Dessa forma, houve uma sensível melhoria da qualidade das inspeções, refletindo num aumento nas notificações oficiais e, por conseguinte, uma redução do número de expedição de alvarás sanitários. No ano de 2010 houve a melhoria nos serviços de fiscalização e os estabelecimentos avaliados tiveram que atender às normas vigentes, refletindo, assim, no aumento de expedições de alvarás sanitários (Figura 1-e).

Salienta-se que a melhoria dos serviços de inspeção sanitária prestados está diretamente relacionada às condições dos serviços de fiscalização pública, além da capacitação e/ou educação continuada de todos os profissionais envolvidos nos serviços de alimentação. Com base nas informações obtidas verificou-se que a qualidade dos serviços prestados pelo setor de alimentos da VISA no município de Santa Maria, RS, está em crescente evolução.

\section{COMITÊ DE ÉTICA E BIOSSEGURANÇA}

O presente estudo foi submetido e aprovado no comitê de ética em pesquisa com seres humanos do Centro Universitário Franciscano (UNIFRA) sob protocolo CEP/ UNIFRA 383.2010.2.

\section{REFERÊNCIAS}

AKUTSU, R. C. et al. Adequação das boas práticas de fabricação em serviços de alimentação. Revista de Nutrição, v.18, n.3, p.419-427, 2005. Disponível em: <http://repositorio.bce.unb.br/ bitstream/10482/2113/1/ARTIGO_AdequacaoBoasPraticas.pdf>. Acesso em: 16 mai. 2012.

ASSOCIAÇÃO BRASILEIRA DAS EMPRESAS DE REFEIÇÕES COLETIVAS (ABERC). Manual ABERC de Práticas de Elaboração e Serviço de Refeições para Coletividades. 9 ed. São Paulo: ABERC, 2009. 221p.

ABREU, E. S. et al Gestão de unidades de alimentação e nutrição: um modo de fazer. 2 ed. São Paulo: Metha, 2011. 360p.

BARBOSA NETA, R. X. et al. Análise dos perigos e pontos críticos de controle durante o preparo da alface servida no restaurante universitário da UFRN. Revista Higiene Alimentar, v. 18, n. 126-127, p. 36-42, 2004.

BENEVIDES, C. M. J.; LOVATTI, R. C. C. Segurança alimentar em estabelecimentos processadores de alimentos. Revista Higiene Alimentar, v. 18, n. 125, p. 24-27, 2004. 
BRASIL. Resolução RDC n 216, de 15 de setembro de 2004. Dispõe sobre Regulamento Técnico de Boas Práticas para Serviços de Alimentação. Ministério da Saúde. Agência Nacional de Vigilância Sanitária (ANVISA). Diário Oficial da União, 16 de setembro de 2004. Disponível em: <http://portal.anvisa.gov.br/ wps/wcm/connect/aa0bc300474575dd83f2d73fbc4c6735/ RDC_N_216_DE_15_DE_SETEMBRO_DE_2004.pdf?MOD= AJPERES>. Acesso em: 17 mai. 2012.

CARDOSO, R. C. V. et al. Unidades de alimentação e nutrição nos campi da Universidade Federal da Bahia: um estudo sob a perspectiva do alimento seguro. Revista de Nutrição, v. 18, n. 5, p. 669-680, 2005. Disponível em: <http://www.scielo.br/ pdf/rn/v18n5/a10v18n5.pdf>. Acesso em: 17 mai. 2012.

CASTRO, F. T. Restaurantes do tipo self-service: análise dos aspectos sanitários e dos manipuladores de estabelecimentos localizados nos shoppings centers da Cidade do Rio de Janeiro RJ, 2007. 105p. Dissertação (Mestrado em Ciência e Tecnologia de Alimentos) Universidade Federal do Rio de Janeiro, Seropédica, RJ. Disponível em: <http://www.ufrrj.br/posgrad/ ppgcta/tesesedissertacoes/dissertacoes/D-239.pdf>. Acesso em: 17 mai.2012.

DAMASCENO, K.S.F.S.C. et al. Condições higênico-sanitárias de "self-services" do entorno da UFPE e das saladas cruas por eles servidas. Revista Higiene Alimentar, v.16, n.102/103, p.74-78, 2002.

DESCHAMPS, C. et al. Avaliação higiênico-sanitária de cozinhas industriais instaladas no município de Blumenau, SC. Revista Higiene Alimentar, v. 17, n.112, p. 12-15, 2003.

GERMANO, P. M. L.; GERMANO, M. I. S. Higiene e Vigilância Sanitária dos Alimentos. 4 ed. São Paulo: Manole. 2011, 1088p.

GOLLUCKE, A. P. B. et al. Avaliação das condições higiênicosanitárias de restaurantes self-service de um município da baixada santista. Revista Higiene Alimentar, v.17, n. 104/105, p.84$85,2003$.

GUIMARÃES, S. L.; FIGUEIREDO, E. L. Avaliação das condições higiênico-sanitárias de panificadoras localizadas no município de Santa Maria do Pará-PA. Revista Brasileira de Tecnologia Agroindustrial, v.4, n.2, p.198-206, 2010. Disponível em: <http://revistas.utfpr.edu.br/pg/index.php/rbta/ article/view/633/589>. Acesso em: 17 mai. 2012.

MACHADO, A. D. et al. Condições higiênico-sanitárias nos serviços de alimentação de Organizações Não Governamentais de Toledo/ PR. Nutrire: Revista da Sociedade Brasileira Alimentação e Nutrição, v. 34, n. 3, p. 141-151, 2009. Disponível em: <http:/ /www.sban.com.br/revistas/NUTRIRE\%20v34\%20n3\%20\%20COMPLETO.pdf>. Acesso em: 17 mai. 2012.
MELLO, A. G. de. et al. Conhecimento dos manipuladores de alimentos sobre boas práticas nos restaurantes públicos populares do Estado do Rio de Janeiro. Brazilian Journal of Food Technology, v. 13, n. 1, p. 60-68, 2010. Disponível em: <http:/ /bj.ital.sp.gov.br/artigos/html/busca/PDF/v13n1405a.pdf >. Acesso em: 17 mai. 2012.

MEZOMO, I. Os serviços de alimentação: planejamento e administração. 4. ed. São Paulo: Manole, 2002. 432p.

MIRANDA, L. K. et al. Panos de prato e mãos de manipuladores: avaliação das condições higiênico-sanitárias. Revista Higiene Alimentar, v. 16, n. 102-103, p. 51-58, 2002.

MURMANN, L. et al. Qualidade do armazenamento de alimentos em estabelecimentos comerciais da cidade de Santa Maria, RS. Revista Higiene Alimentar, v. 19, n. 147, p. 29-33, 2005.

PILLA, C. S. Perfil das denúncias recebidas pelo programa de alimentos da Vigilância Sanitária de Viamão/RS. 2009. 45p. Monografia (Graduação em Medicina Veterinária) Universidade Federal do Rio Grande do Sul, Porto Alegre, RS. Disponível em: <http://www.lume.ufrgs.br/bitstream/handle/ 10183/22912/000734571.pdf?sequence=1>. Acesso em: 17 mai.2012.

PRAXEDES, P. C. G. Aspectos da qualidade higiênicosanitária de alimentos consumidos e comercializados na cidade de São Remo. 2003. 120 f. Dissertação (Mestrado em Epidemiologia Experimental e Aplicada ao Controle das Zoonoses) - Universidade de São Paulo, São Paulo, 2003.

RIO GRANDE DO SUL. Portaria n 78/2009. Aprova a Lista de Verificação em Boas Práticas para Serviços de Alimentação,aprova Normas para Cursos de Capacitação em Boas Práticas para Serviços de Alimentação e dá outras providências. Secretaria da Saúde do Estado do Rio Grande do Sul. Diário Oficial/RS, 30 de janeiro de 2009. Disponível em: <http://www.saude.rs.gov.br/ dados/1293546540816portaria\%2078-09\%20pag.\%2035.pdf $>$. Acesso em: 17 mai. 2012.

SILVA, E. A. Manual de Controle Higiênico-Sanitário em Serviços de Alimentação. 6 ed. São Paulo: Varella. 2010. 623p.

STORCK, C. R.; DIAS, M. A. M. F. Monitoramento da temperatura de preparações quentes e frias em restaurantes selfservices na zona urbana de Santa Maria. Revista Nutrição em Pauta, v. 11, n. 59, p. 31- 35, 2003. Disponível em: <http:// www.nutricaoempauta.com.br/busca.php?cod_revista=59>. Acesso em: 17 mai. 2012.

WALKER, E. et al. Food handler's hygiene in small food businesses. Food Control, v. 14, n. 5, p. 339-343, 2003. Disponível em: <http://ac.els-cdn.com/S0956713502001019/ 1 -s2.0-S0956713502001019-main.pdf?_tid=e400800c99 66b6418ceb1419d57b04e8\&acdnat $=1337266151 \_330 \mathrm{fe} 87$ ded6a13c48505cc3c3c033267>. Acesso em: 17 mai 2012. 